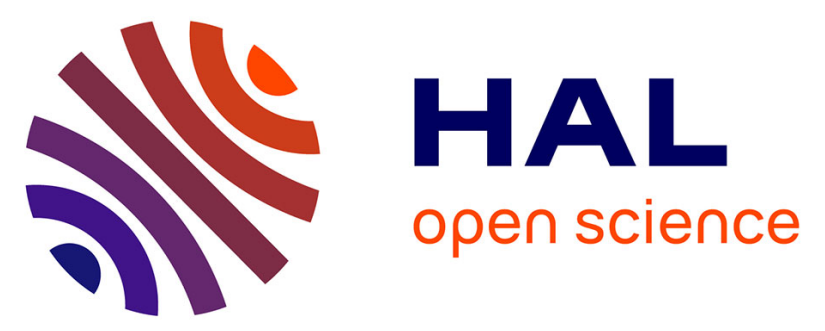

\title{
Glassy States in Asymmetric Mixtures of Soft and Hard Colloids
}

Domenico Truzzolillo, Daniela Marzi, John Marakis, Barbara Capone, Manuel

Camargo, Abdul Munam, Firmin Moingeon, Mario Gauthier, Christos Likos,

Dimitris Vlassopoulos

\section{To cite this version:}

Domenico Truzzolillo, Daniela Marzi, John Marakis, Barbara Capone, Manuel Camargo, et al.. Glassy States in Asymmetric Mixtures of Soft and Hard Colloids. Physical Review Letters, 2013, 111 (20), pp.208301. 10.1103/PhysRevLett.111.208301 . hal-00909433

\section{HAL Id: hal-00909433 \\ https://hal.science/hal-00909433}

Submitted on 4 Jun 2021

HAL is a multi-disciplinary open access archive for the deposit and dissemination of scientific research documents, whether they are published or not. The documents may come from teaching and research institutions in France or abroad, or from public or private research centers.
L'archive ouverte pluridisciplinaire HAL, est destinée au dépôt et à la diffusion de documents scientifiques de niveau recherche, publiés ou non, émanant des établissements d'enseignement et de recherche français ou étrangers, des laboratoires publics ou privés. 


\title{
Glassy States in Asymmetric Mixtures of Soft and Hard Colloids
}

\author{
Domenico Truzzolillo, ${ }^{1,2,3}$ Daniela Marzi,${ }^{4}$ John Marakis, ${ }^{1}$ Barbara Capone, ${ }^{4}$ Manuel Camargo, ${ }^{5}$ Abdul Munam, ${ }^{6}$ \\ Firmin Moingeon, ${ }^{6}$ Mario Gauthier, ${ }^{6}$ Christos N. Likos, ${ }^{4}$ and Dimitris Vlassopoulos ${ }^{1}$ \\ ${ }^{1}$ FO.R.T.H., Institute of Electronic Structure and Laser, and Department of Materials Science \& Technology, \\ University of Crete, GR-71003 Heraklion, Greece \\ ${ }^{2}$ Université Montpellier 2, Laboratoire Charles Coulomb UMR 5221, F-34095 Montpellier, France \\ ${ }^{3}$ CNRS, Laboratoire Charles Coulomb UMR 5221, F-34095 Montpellier, France \\ ${ }^{4}$ Faculty of Physics, University of Vienna, Boltzmanngasse 5, A-1090 Vienna, Austria \\ ${ }^{5}$ Centro de Investigaciones en Ciencias Básicas, Universidad Antonio Nariño, Kra 3 Este 47a-15, Bogotá, Colombia \\ ${ }^{6}$ Department of Chemistry, University of Waterloo, Waterloo, Ontario N2L 3G1, Canada
}

(Received 3 July 2013; published 14 November 2013)

\begin{abstract}
By employing rheological experiments, mode coupling theory, and computer simulations based on realistic coarse-grained models, we investigate the effects of small, hard colloids on the glassy states formed by large, soft colloids. Multiarm star polymers mimic hard and soft colloids by appropriately varying the number and size of their arms. The addition of hard colloids leads, depending on their concentration, to either melting of the soft glass or the emergence of two distinct glassy states. We explain our findings by depletion of the colloids adjacent to the stars, which leads to an arrested phase separation when the repulsive glass line meets the demixing binodal. The parameter-free agreement between experiment, theory, and simulations suggests the generic nature of our results and opens the route for designing soft-hard colloidal composites with tunable rheology.
\end{abstract}

DOI: 10.1103/PhysRevLett.111.208301

PACS numbers: 82.70.Dd, 64.70.kj, 83.80.Rs

Suspensions of colloidal particles are ubiquitous in nature and represent an ever stimulating and technologically challenging field of research [1]. The majority of the work relating to the dynamics and rheology of colloids has been performed with the simplest hard sphere systems, both experimentally and theoretically [2,3]. Indeed, in a breakthrough study [4] it was demonstrated that added nonadsorbing polymers, smaller than the colloids, control the rich morphology diagram of colloid-polymer mixtures and its rheological consequences primarily via depletion. In general, colloidal mixtures exhibit a variety of phases and kinetic states from colloidal gas to liquid, crystal, repulsive, and attractive glass and gel [5].

These important developments prompted the question of the effects of soft interactions on the macroscopic properties of the colloids [6,7]. Star polymers have been explored in detail, since they exemplify stable, long hairy particles. At high concentrations, they deform but also interpenetrate via their grafted arms, as reflected by their effective interactions: their tunability at the molecular level allows repulsive pair potentials ranging from ultrasoft to hard as their functionality (number of arms) $f_{S}$ changes from typically below 30 to above 400 [8]. The very same deformability also determines their purely entropic interactions with planar or curved hard walls [9]. Stars with functionalities $f_{S} \gtrsim 50$ form glassy states at large volume fractions $[7,10]$. Mixtures of star polymers and smaller, linear polymers have shown great richness in metastable states, controlled by the osmotic pressure of the latter, and leading to star shrinkage and depletion $[11,12]$. Star polymers with
$f_{S} \leq 32$ were also mixed with larger hard spheres and the phase behavior of the mixtures was examined experimentally and theoretically [13]. However, the inverse case, in which hard spheres are used as depletants for deformable, glass-forming stars has not been addressed to date.

In this Letter, we address the formation of multiple glassy states in soft-hard colloidal mixtures composed of large stars and small hard spherelike depletants. To ensure purely entropic interactions, we used stars of the same chemistry and drastically different functionalities. The hard colloids were stars with very short chains, expected to be fully stretched in the core portion [14], so that they act as hard spheres (HS). Using rheology, mode-coupling theory (MCT) and molecular dynamics (MD) simulations, we observe vitrification of the soft star glass, melting upon the addition of colloidal spheres and subsequent arrested phase separation as the repulsive glass line crosses the binodal.

Polybutadiene star polymers (1,4 addition) of $f_{S}=214$ (soft sphere) and $f_{C}=1109$ (hard sphere), and respective arm molar masses 67000 and $1270 \mathrm{~g} / \mathrm{mol}$, were synthesized anionically. The polydispersity indexes (PDI) and the molar fraction of contaminants (MFC) have been measured via size exclusion chromatography: $\mathrm{PDI}=1.07$ and $\mathrm{MFC}=0.12$ for the soft spheres, whereas $\mathrm{PDI}=1.11$ and $\mathrm{MFC}=0.036$ for the hard spheres. Details are given in Refs. [15,16] and in the Supplemental Material [17]. To express the concentrations in terms of the number densities of the star components and colloid components $\left(\rho_{S}\right.$ and $\rho_{C}$, respectively), we employ the effective corona diameter of the stars $\sigma_{S}$ as the unit of length. The star-to-HS 
hydrodynamic size ratio is $\xi=4$ and the nominal glass transition concentrations for the stars and HS are $\rho_{S} \sigma_{S}^{3}=$ $0.339 \pm 0.002$ and $\rho_{C} \sigma_{S}^{3}=10.75 \pm 0.7$. Accordingly, the pure star solutions were investigated at number densities $\rho_{S} \sigma_{S}^{3}=0.342,0.347,0.368$, and 0.421 , in which the samples are glassy, and colloidal spheres of various concentrations have been added to analyze the ensuing rheology of the mixture. Here, we focus on the effects of adding HS to stars at $\rho_{S} \sigma_{S}^{3}=0.342$, where more than one glassy state as well as melting of the mixture can be unambiguously distinguished.

In the absence of HS, the concentrated star solution with $\rho_{S} \sigma_{S}^{3}=0.342$ exhibits features typical of glassy behavior [Fig. 1(a)]: both the storage $\left(G^{\prime}\right)$ and loss $\left(G^{\prime \prime}\right)$ moduli are only weakly frequency dependent, with $G^{\prime \prime}$ exhibiting a broad minimum, $G^{\prime}(\omega)>G^{\prime \prime}(\omega)$ over four decades of frequency, while being also time dependent due to aging [7,17].

While maintaining the star density fixed at $\rho_{S} \sigma_{S}^{3}=$ 0.342 , the addition of HS colloids induces remarkable softening of the initial glassy suspension, and eventually a transition to the ergodic liquid state above a certain HS volume fraction $\left(\rho_{C} \sigma_{S}^{3}>0.029\right)$ is observed, Fig. 1(b). This is also confirmed by the absence of aging [7,17]. For even higher HS fractions $\left(\rho_{C} \sigma_{S}^{3} \geq 1.0311\right)$, shown in Fig. 1(c), the mixture undergoes a reentrant transition to another solidlike state which, with the help of MCT, is attributed to arrested phase separation; the latter is corroborated by two facts: (i) at the reentrance the plateau modulus $G_{p}$ is nearly identical to that of the original repulsive glass (see Fig. 2), and (ii) there is no visual evidence of this dense sample becoming turbid. This suggests a scenario of a purely repulsive glass regime up to the reentrant line, an interpretation supported by the theoretical analysis. Note that if the reentrant state were an attractive glass, its storage modulus would have been much higher as compared to that of the repulsive glass [18]. We also observe in Fig. 2 that upon further increasing the concentration of HS colloids in the reentrant regime, the modulus eventually increases by more that one decade. It is tempting to interpret this increase as a third transition, but this requires additional experimental and theoretical work, which are currently under way. Turning to the intermediate ergodic regime, the characteristic time of the liquid (extracted from the terminal crossover of the moduli) varies by several decades: it first decreases with $\rho_{C}$ as we move away from the repulsive glass due to depletion, and then goes through a minimum and increases as the reentrance is approached (Fig. 2). The rheological yielding transition was examined with dynamic strain sweeps at different frequencies (here the $10 \mathrm{rad} / \mathrm{s}$ results are discussed). While a single yield point could be identified in the initial repulsive glassy phase [Fig. 1(a), inset] and no yielding was observed in the liquid state [Fig. 1(b), inset], the reentrant state exhibited a two-step yield process [Fig. 1(c), inset] for $\rho_{C} \sigma_{S}^{3}<3.53$. Beyond this value the single yielding behavior was recovered along
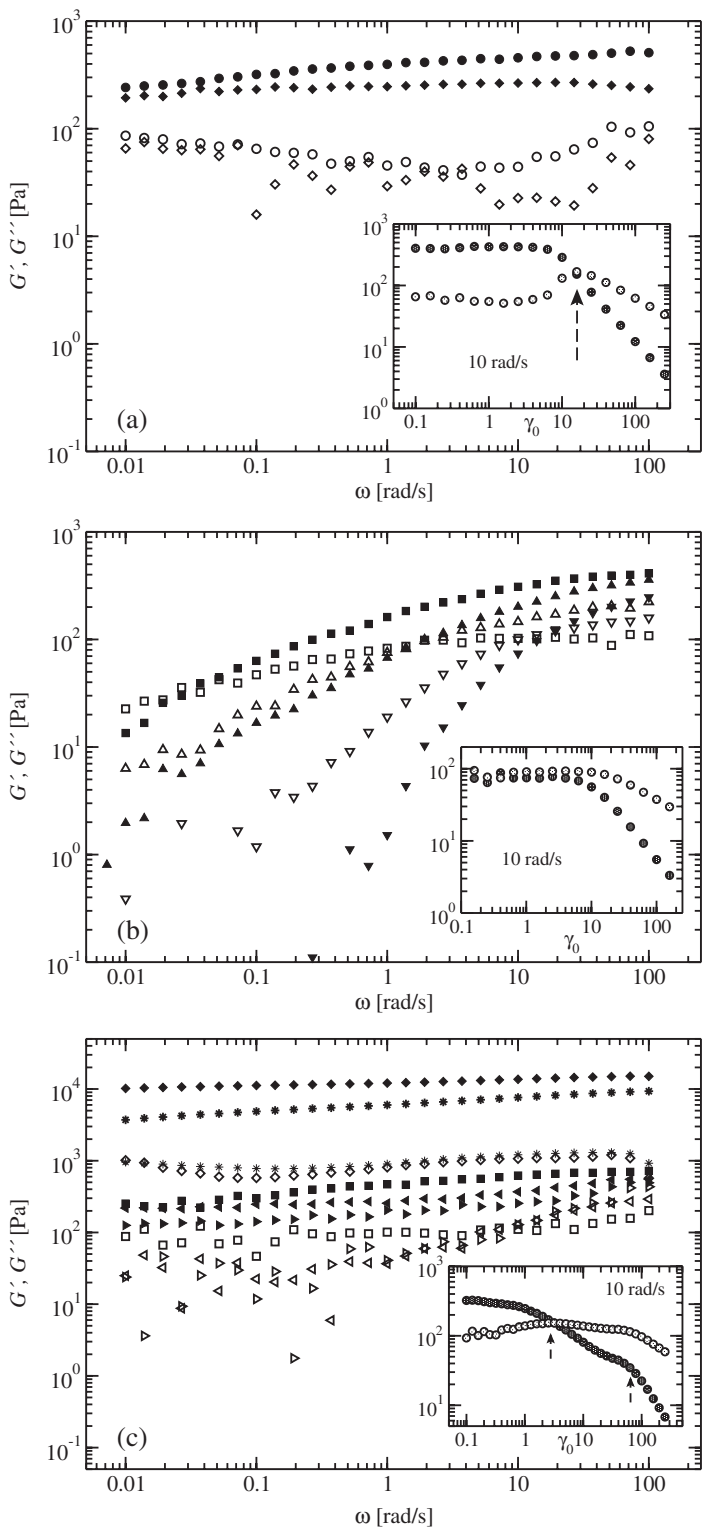

FIG. 1. Linear viscoelastic spectra for HS-star mixtures at $\Phi_{S}=1.625\left(\rho_{S} \sigma_{S}^{3}=0.342\right) . G^{\prime}$ and $G^{\prime \prime}$ are shown with full and empty symbols, respectively. (a) Repulsive glass: $\rho_{C} \sigma_{S}^{3}=0$ (circles) and $\rho_{C} \sigma_{S}^{3}=0.0147$ (diamonds). Inset: Dynamic strain sweep for $\rho_{C} \sigma_{S}^{3}=0$ at $10 \mathrm{rad} / \mathrm{s}$. The arrow indicates the yield point. (b) Liquid: $\rho_{C} \sigma_{S}^{3}=0.1473$ (squares); $\rho_{C} \sigma_{S}^{3}=0.2946$ (up triangles); $\rho_{C} \sigma_{S}^{3}=0.4419$ (down triangles). Inset: Dynamic strain sweep for $\rho_{C} \sigma_{S}^{3}=0.4419$ at $10 \mathrm{rad} / \mathrm{s}$. (c) Reentrant glass: $\rho_{C} \sigma_{S}^{3}=1.1784$ (squares); $\rho_{C} \sigma_{S}^{3}=1.7676$ (left triangles); $\rho_{C} \sigma_{S}^{3}=3.5352$ (right triangles); $\rho_{C} \sigma_{S}^{3}=5.892$ (stars); $\rho_{C} \sigma_{S}^{3}=$ 7.070 (diamonds). Inset: Dynamic strain sweeps for $\rho_{C} \sigma_{S}^{3}=$ 1.7676, showing $G^{\prime}$ (dark circles) and $G^{\prime \prime}$ (light circles). The arrows mark the yield points: two-step yielding occurs in the arrested phase separated mixtures.

with an increase in the modulus. Double yielding is thought of as reflecting two constraining length scales (say, bonds and cages) which occur in attractive glasses [18] or arrested phase separating systems alike. It is tempting to interpret the 


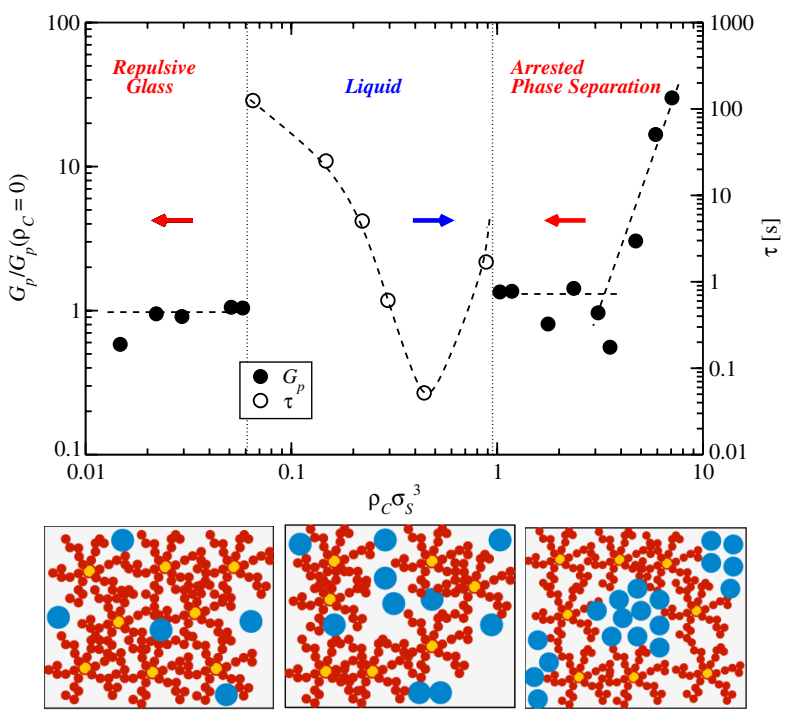

FIG. 2 (color online). The plateau moduli of the arrested states (closed circles, left vertical axis) and the relaxation times of the ergodic phases (open circles, right vertical axis) observed for star polymer density $\rho_{S} \sigma_{S}^{3}=0.342$ as a function of the colloid density $\rho_{C} \sigma_{S}^{3}$. The three cartoons show, from left to right, sketches of the repulsive glass, the liquid, and the arrested phase separation state.

observed increase of the modulus as a third transition but this requires additional experimental and theoretical work, which is currently under way.

Figure 3(a) assembles all experimental data in the form of a morphology diagram where the different rheological states are indicated for varying fractions of star and the added HS depletant particles. The vertical arrow indicates the onset of the glassy star regime at $\rho_{C}=0$. Thereafter, for $\rho_{S} \sigma_{S}^{3}=0.342$, which is the most extensively studied mixture, we observe the transformation from a repulsive glass to a liquid and eventually to a reentrant arrested state, as a result of the depletion effect of the added HS. Finally, for larger $\rho_{S}$ we find glasses and glass-glass transitions with the glass at larger $\rho_{C}$ having larger moduli, consistently with the presence of attraction in the system cages.

The theoretical analysis was based upon a coarse-grained picture of the binary mixture. Thereby effective interaction potentials $V_{i j}(r), i, j=S, C$, acting between the centers of two particles separated by distance $r$ could be employed. For the star-star interaction $V_{S S}(r)$, the potential introduced in Ref. [8] was employed, which features a crossover from a logarithmic divergence at small $r$ into a Yukawa form for large $r$ at the corona diameter $\sigma_{S}$. Comparisons with experiments $[8,11,19,20]$ have shown that $\sigma_{S} \cong R_{h}^{S} \cong 4 R_{g} / 3, R_{g}$ being the radius of gyration of the stars [21]. $V_{C C}(r)$ was modeled as a hard sphere potential for a diameter $\sigma_{C}=2 R_{C}$, a choice justified by the ultrahigh functionality of the hardlike stars. For $V_{S C}(r)$ we employed the recently developed corresponding potentials [9] for star functionality $f_{S}=214$ and $\xi=4$, as in the experiments. We used two-component
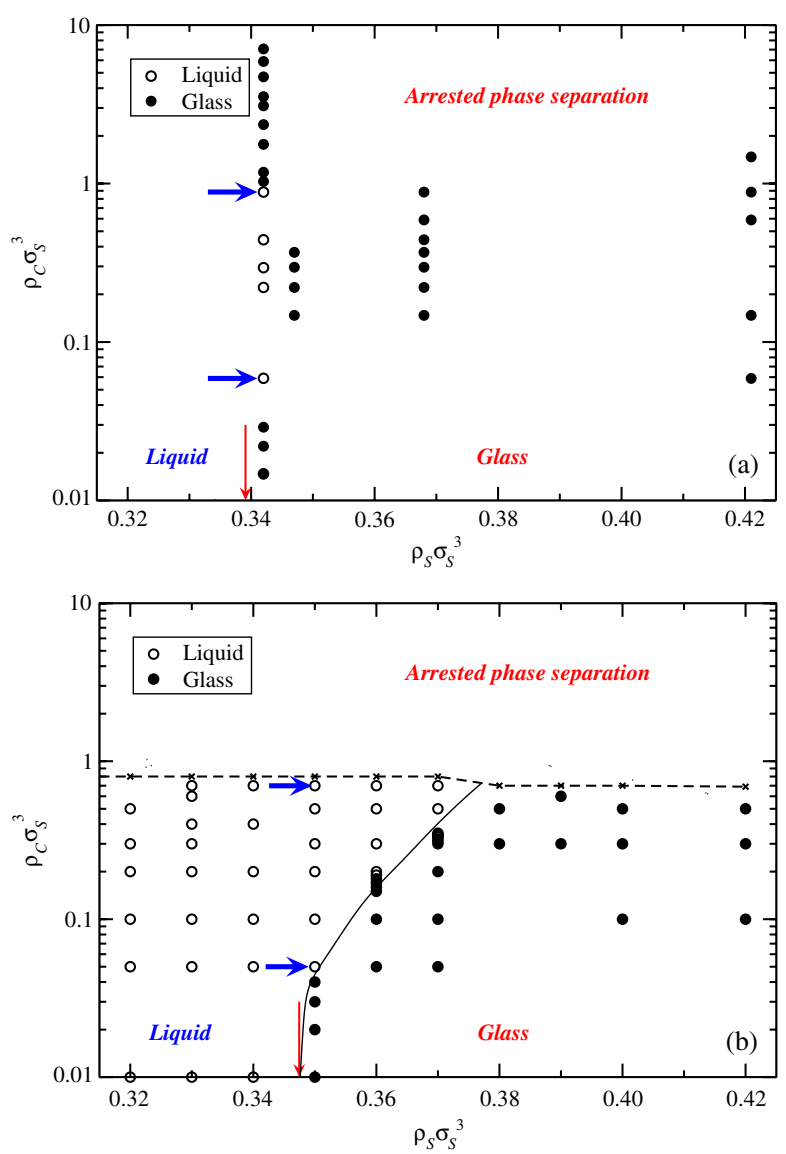

FIG. 3 (color online). (a) The experimental state diagram of star polymer-colloidal mixtures with $\xi=4$ and star functionality $f_{S}=214$. The vertical arrow denotes the star polymer density at which a star solution arrests in the experiment in the absence of colloids and the horizontal arrows denote the points where the system melts and revitrifies. (b) The MCT-phase diagram of the same system. The dashed line denotes the locus of points for which integral equations fail to converge due to a demixing phase transition, whereas the hand-drawn solid line separates the region of the liquid and the repulsive glass. Arrows and circles as in panel (a).

integral equation theories with the Rogers-Young closure [22] to calculate the partial structure factors for mixtures with arbitrary density and composition. Their validity was confirmed by the excellent agreement with Monte Carlo simulations for the coarse-grained mixtures at selected points.

The locus of ideal glass transition points on the $\left(\rho_{S}, \rho_{C}\right)$ plane was determined by employing the one-component version of the MCT [20,23], in which the structure factor $S(k)$ of the stars is the input for the determination of their nonergodicity factor $f(k)$. The latter vanishes for an ergodic liquid and it is nonzero for a glass. Inherent in this one-component approach is the assumption that within the glassy state of the stars, the small colloidal particles remain ergodic; were this not to be the case, then the full, two-component version of the MCT should be employed 
$[19,23]$. This assumption was fully confirmed by our MD simulations [17].

Figure 4 shows the dependence of the nonergodicity factor for a star solution at density $\rho_{S} \sigma_{S}^{3}=0.36$, for which the pure system is glassy, on the density of the added colloids. Upon addition of a sufficient amount of HS particles $\left(\rho_{C} \sigma_{S}^{3} \geq 0.19\right)$, the glass melts. The physical mechanism responsible for this transition lies in the fact that the small colloids act as depletants for the large stars-a situation specular to the usual colloid or polymer case, in which the small polymers deplete the big colloids $[4,13,24-26]$. In contrast to the latter case, in which the depletion attraction takes the form of an Asakura-Oosawa or square-well potential [24], for the system at hand the colloid-induced depletion is superimposed on a soft repulsion between the stars. Thus, at moderate colloid concentrations, it has initially the effect of reducing the repulsions, leading to melting of the glass [11,19]. Upon further addition of colloid, net attractions between the stars appear [17], which drive the system towards a demixing phase separation into a star- and a colloid-rich phase, as already predicted in Refs. $[9,27]$. This manifests itself in the integral equation approach by the fact that all structure factors develop increasingly high peaks at $k=0$, and convergence of the integral equations can no longer be achieved. The locus of points for which the solution is lost, which can be loosely identified with the demixing line of the system, is denoted in Fig. 3(b) by the dashed line. This figure presents a compilation of the MCT results and it should be directly compared to its experimental counterpart, Fig. 3(a). Quantitative and parameter-free agreement can be seen between experiment and theory by comparing Fig. 3(a) with Fig. 3(b) regarding

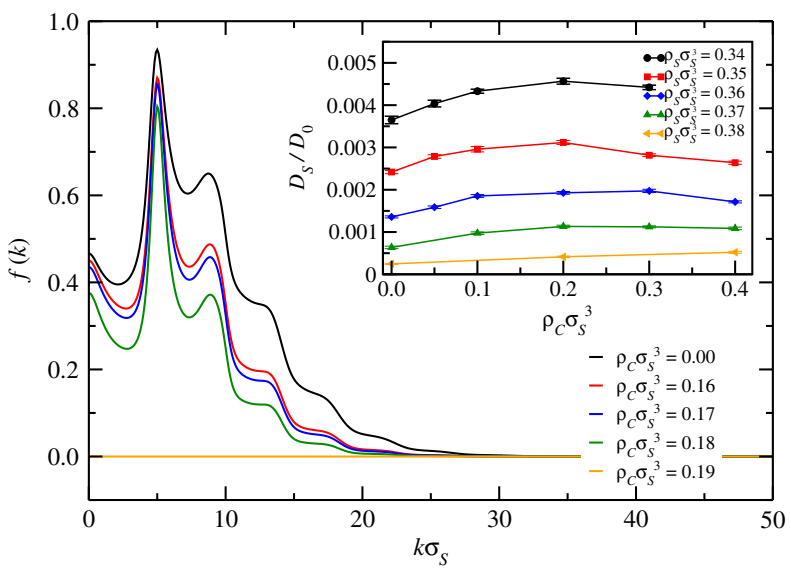

FIG. 4 (color online). The dependence of the star nonergodicity factor $f(k)$ for a star polymer solution at density $\rho_{S} \sigma_{S}^{3}=0.36$ on the density of added colloids, as indicated in the legend. Between $\rho_{C} \sigma_{S}^{3}=0.18$ and $\rho_{C} \sigma_{S}^{3}=0.19, f(k)$ drops abruptly to zero, indicating an ideal glass-to-liquid transition. Inset: the long-time diffusion constant $D_{S}$ of the stars, normalized with $D_{0}=\sqrt{k_{\mathrm{B}} T \sigma_{S}^{2} / m_{S}}$, as a function of the density of the colloidal additives and for various star densities as indicated in the legend. the glass-to-liquid transition for the value of $\rho_{S}$ lying most closely to the glass transition in the absence of colloids, denoted by the horizontal arrows there.

For higher star densities, theory predicts gradual shrinkage of the liquid regime, which forms an intruding pocket into the glassy region. The extent of this intrusion is overestimated by theory. The reentrant glass observed in experiments can arise from the intervention of the demixing binodal, thus rendering the high- $\rho_{C}$ glass as the result of an arrested phase separation. The latter has been extensively discussed in the literature in conjunction with gelation, and in all cases within the context of colloid-polymer mixtures with very sharp and short-range attractions caused by the polymers depleting the colloids [25,26,28-32]. In that case, it has been shown [29,32] that the attractive glass line intersects the binodal on the high-density side of the glass former. Here, it is the repulsive glass line that meets the binodal on the high-density side of the star-polymer glass former. However, as the binodal is crossed, the ensuing phase separation becomes arrested in the continuation of the glass line within the demixed region and the system arrests into a high-star concentration glass. This increase in concentration compensates for the reduced repulsions and leads to a glass whose modulus is essentially the same as that of the original one. Deeply inside the binodal, i.e., for even higher values of $\rho_{C}$, depletion attractions are strong and can lead to arrest into an attractive glass with much higher values of the modulus (Fig. 2). The high- $\rho_{S}$ region above the demixing line in Fig. 3(b) thus corresponds to arrested states, consistently with the experimental findings for reentrant glasses. It is not amenable to MCT analysis, though, since no structural data are available there, due to the loss of solutions of the integral equations.

We have assumed in our MCT analysis that the colloidal additives remain ergodic within the star glass; it is not obvious whether this assumption is correct; hence, its validity has to be proven. Zaccarelli et al. [23] have shown that in a binary mixture the long-time dynamics are determined by the short-time mobility ratio $\alpha$ between the large and the small components. For Brownian particles, as for the case at hand, this is set by the hydrodynamic size ratio: $\alpha=\xi^{-1}=0.25$. The assumption of colloidal ergodicity has been validated via MD simulations [17]. Indeed, the mean-square displacements of the colloids showed typical diffusive behavior. On the contrary, the mean-square displacement curves for the stars displayed a clear plateau at the crossover from the ballistic to the diffusive regime, which shrunk upon the addition of colloids [17]. The measured long-time diffusion constants $D_{S}$ for the stars are summarized in the inset of Fig. 4. Consistently with the MCT results, the addition of colloids first accelerates the star dynamics and then slows them down again as the colloidal concentration is increased, whereas phase separation sets in at even higher colloidal densities. This nonmonotonic behavior is consistent with glass reentrance and 
with that of the relaxation time of the fluid shown in Fig. 2, middle panel.

In conclusion, we have shown by combining experiments, theory, and simulation that the addition of small, hard colloidal additives to a structural glass formed by large, soft colloids brings forward a wealth of novel, tunable rheological states, including a melting to an ergodic liquid and new states that appear due to an interplay between the structural arrest line of the repulsive glass and macroscopic demixing. The star functionality and the size ratio represent the natural, physical parameters to adjust in order to steer the rheology of the mixtures. The quantitative, parameter-free agreement between the experiments, simulations, and theory shown in this Letter offers a strong basis for the extension of our investigations to other functionalities and size ratios in the future.

This work has been supported by the EU (ITNCOMPLOIDS Grant No. 234810) and by the J. S. Latsis Foundation (Grant No. 0839-2012).

[1] W. Russel, D. Saville, and W. Schowalter, Colloidal Dispersions (Cambridge University Press, London, 1989).

[2] J. Mewis and N.J. Wagner, Colloidal Suspension Rheology (Cambridge University Press, London, 2012).

[3] P. N. Pusey, in Liquids Freezing and the Glass Transition, Proceedings of the Les Houches Summer School, Session LI, edited by J. Hansen, D. Levesque, and J. Zinn-Justin (North-Holland, Amsterdam, 1991), pp. 763-942.

[4] K. N. Pham, A. M. Puertas, J. Bergenholtz, S. U. Egelhaaf, A. Moussaïd, P. N. Pusey, A. B. Schofield, M. E. Cates, M. Fuchs, and W. C. K. Poon, Science 296, 104 (2002).

[5] A. Imhof and J. K. G. Dhont, Phys. Rev. Lett. 75, 1662 (1995).

[6] C. N. Likos, Phys. Rep. 348, 267 (2001).

[7] D. Vlassopoulos and G. Fytas, Adv. Polym. Sci. 236, 1 (2010).

[8] C. N. Likos, H. Löwen, M. Watzlawek, B. Abbas, O. Jucknischke, J. Allgaier, and D. Richter, Phys. Rev. Lett. 80, 4450 (1998).

[9] D. Marzi, C. N. Likos, and B. Capone, J. Chem. Phys. 137, 014902 (2012).

[10] G. Foffi, F. Sciortino, P. Tartaglia, E. Zaccarelli, F. Lo Verso, L. Reatto, K. A. Dawson, and C. N. Likos, Phys. Rev. Lett. 90, 238301 (2003).

[11] E. Stiakakis, D. Vlassopoulos, C. N. Likos, J. Roovers, and G. Meier, Phys. Rev. Lett. 89, 208302 (2002).
[12] D. Truzzolillo, D. Vlassopoulos, and M. Gauthier, Macromolecules 44, 5043 (2011).

[13] J. Dzubiella, A. Jusufi, C. N. Likos, C. von Ferber, H. Löwen, J. Stellbrink, J. Allgaier, D. Richter, A. Schofield, P. Smith et al., Phys. Rev. E 64, 010401(R) (2001).

[14] M. Daoud and J. P. Cotton, J. Phys. (Paris) 43, 531 (1982).

[15] J. Roovers, L. Zhou, P. M. Toporowski, M. van der Zwan, H. Iatrou, and N. Hadjichristidis, Macromolecules 26, 4324 (1993).

[16] M. Gauthier and A. Munam, Macromolecules 43, 3672 (2010).

[17] See Supplemental Material at http://link.aps.org/ supplemental/10.1103/PhysRevLett.111.208301 for details of the rheological experiments and technical aspects of the theory and simulation.

[18] K. N. Pham, G. Petekidis, D. Vlassopoulos, S. Egelhaaf, W. C. K. Poon, and P. N. Pusey, J. Rheol. 52, 649 (2008).

[19] C. Mayer, E. Zaccarelli, E. Stiakakis, C. N. Likos, F. Scortino, A. Munam, M. Gauthier, N. Hadjichristidis, H. Iatrou, P. Tartaglia et al., Nat. Mater. 7, 780 (2008).

[20] C. Mayer, F. Sciortino, C. N. Likos, P. Tartaglia, H. Löwen, and E. Zaccarelli, Macromolecules 42, 423 (2009).

[21] A. Jusufi, M. Watzlawek, and H. Löwen, Macromolecules 32, 4470 (1999).

[22] F. J. Rogers and D. A. Young, Phys. Rev. A 30, 999 (1984).

[23] E. Zaccarelli, H. Löwen, P. P. F. Wessels, F. Sciortino, P. Tartaglia, and C. N. Likos, Phys. Rev. Lett. 92, 225703 (2004).

[24] K. Dawson, G. Foffi, M. Fuchs, W. Götze, F. Sciortino, M. Sperl, P. Tartaglia, T. Voigtmann, and E. Zaccarelli, Phys. Rev. E 63, 011401 (2000).

[25] A. M. Puertas, M. Fuchs, and M. E. Cates, J. Phys. Chem. B 109, 6666 (2005).

[26] A. M. Puertas, M. Fuchs, and M.E. Cates, J. Phys. Condens. Matter 19, 205140 (2007).

[27] N. A. Mahynski and A.Z. Panagiotopoulos, J. Chem. Phys. 139, 024907 (2013).

[28] A. Stradner, H. Sedwick, F. Cardinaux, W. Poon, S. U. Egelhaaf, and P. Schurtenberger, Nature (London) 432, 492 (2004).

[29] G. Foffi, C. De Michele, F. Sciortino, and P. Tartaglia, Phys. Rev. Lett. 94, 078301 (2005).

[30] E. Zaccarelli, I. Saika-Voivod, S. V. Buldyrev, A. J. Moreno, P. Tartaglia, and F. Sciortino, J. Chem. Phys. 124, 124908 (2006).

[31] F. Cardinaux, T. Gibaud, A. Stradner, and P. Schurtenberger, Phys. Rev. Lett. 99, 118301 (2007).

[32] P. J. Lu, E. Zaccarelli, F. Ciulla, A. B. Schofield, F. Sciortino, and D. A. Weitz, Nature (London) 453, 499 (2008). 